\title{
Prognostic impact of TAZ and $\beta$-catenin expression in adenocarcinoma of the esophagogastric junction
}

\author{
Lidan Sun ${ }^{1,2}$, Fei Chen ${ }^{1}$, Wenna Shi ${ }^{3}$, Lei Qi ${ }^{4}$, Zhongmei Zhao ${ }^{5}$ and Jianping Zhang ${ }^{1,2^{*}}$
}

\begin{abstract}
Background: TAZ is a downstream agent of Hippo signal pathway. $\beta$-catenin is a cell adhesion molecule associated with the invasion and metastasis of carcinomas as well as a critical component of Wnt pathway. TAZ and $\beta$-catenin have long been thought to play a vital role in tumour development and progression. This study aimed to detect expression of TAZ and $\beta$-catenin in adenocarcinoma of the esophagogastric junction (AEG) and explore their clinicopathological significance.
\end{abstract}

Methods: The expression of TAZ and $\beta$-catenin were detected by immunohistochemistry of 135 AEG samples, and analyzed with complete clinicopathological features. Overall survival rates were also calculated using the Kaplan-Meier method. Cox proportional hazard model was performed to assess the prognostic values. 37 normal mucosa and 41 dysplasia samples of esophagogastric junction (EGJ) were studied comparably.

Results: TAZ protein showed a strictly nuclear staining pattern in AEG and dysplasia with IHC. Expression of TAZ was higher in dysplasia and AEG compared with normal mucosa $(P<0.001,0.008)$. The positive expression rate of nuclear $\beta$-catenin was significantly higher in carcinoma and dysplasia than that in normal mucosa $(P<0.001,=0.046)$. Abnormal expression rate of membranous $\beta$-catenin in AEG was significantly higher than that in normal mucosa tissues and dysplasia $(P=0.001,0.002)$. In $A E G$, over expression of TAZ was directly correlated with abnormal nuclear $\beta$-catenin expression $(r=0.298, P<0.001)$ and membranous $\beta$-catenin $(r=0.202, P=0.019)$. Patients with abnormal TAZ or $\beta$-catenin expression of AEG exhibited a shorter overall survival (OS) and lower overall survival rate than those with normal TAZ or $\beta$-catenin expression $(P<0.05)$. In addition, patients with abnormal expression of both TAZ and $\beta$-catenin exhibited worst overall survival. In multivariate survival analysis, abnormal expression of TAZ, TAZ \& $\beta$-catenin (nuclear and membranous) and tumour differentiation were found to be independent prognostic factors related to OS of AEG patients.

Conclusions: Over expression of TAZ was associated with abnormal expression of $\beta$-catenin, which is correlated with poor prognosis of patients with AEG. Abnormal expression of TAZ and TAZ \& $\beta$-catenin (nuclear and membranous) are independent prognostic factors, so targeting TAZ and $\beta$-catenin could prove to be a promising therapeutic strategy for the treatment of AEG.

Virtual Slides: The virtual slide(s) for this article can be found here: http://www.diagnosticpathology.diagnomx.eu/vs/ 2558852841276335

Keywords: TAZ, $\beta$-catenin, Correlation, Adenocarcinoma of the esophagogastric junction, Esophagogastric junction, Prognosis, Immunohistochemistry

\footnotetext{
* Correspondence: jianpingzhang@sdu.edu.cn

'Department of Pathology, Shandong University School of Medicine, 44\#,

Wenhua Xi Road, Jinan, Shandong 250012, People's Republic of China

2Department of Pathology, Qilu Hospital, Shandong University, 107\#,Wenhua

Xi Road, Jinan, Shandong 250012, People's Republic of China

Full list of author information is available at the end of the article
} 


\section{Background}

Adenocarcinoma of the esophagogastric junction (AEG) is defined as carcinoma that crosses the esophagogastric junction (EGJ) line, irrespective of where the tumour epicenter is located, including both distal esophageal and proximal gastric carcinomas [1]. In recent decades, AEG has gradually become a research hotspot for its rising incidence and obscure causes [2,3]. The recognition of AEG is not unanimous for the underlying mechanisms are poorly understood. There are still many controversies in its definition, classification, diagnosis and treatment. Some scientists distinguished patients with EGJ tumours from those with gastric cancer [4], but some treated them as esophageal cancer [5] or gastric tumours [6]. Therefore, this study explores the features of AEG to provide some evidence for clinical diagnosis and treatment.

The transcriptional coactivator with PDZ-binding motif (TAZ), also called WWTR1 (WW-domain containing transcriptional regulator 1), was first reported as a 14-3-3 binding protein [7] which is very similar to Yes-associated protein (YAP). It is one of the downstream agents of Hippo pathway which plays an important role in maintaining organ size and the regulation of cell proliferation and apoptosis $[8,9]$. The core components of Hippo pathway are mammalian STE20-like protein kinase 1/2 [10], large tumour suppressor $1 / 2$ [11] and their adaptor proteins: Salvador homologue 1 [12] and MOB kinase activator respectively. That is a kinase cassette and finally leads to the phosphorylation of TAZ and YAP. Phosphorylation of YAP and TAZ loses their biological activities and stimulates their ubiquitin-mediated proteolysis [13]. Consequently, TAZ losses the ability of interacting with a variety of transcription factors to regulate cell growth differentiation and apoptosis, including Runx2, PPAR, TBX5, TEADs, TTF-1, and PAX3 [14-19].

$\beta$-catenin was originally identified as a membrane component of the cadherin-mediated cell-cell adhesion system and it is now widely recognized as a critical element of the Wnt signal pathway [20]. In the absence of Wnt signals, $\beta$-catenin is located at the plasma membrane, linked to E-cadherin, and functions in cell-cell adhesion. Excess cytoplasmic $\beta$-catenin is sequestered in a protein complex comprised of glycogen synthase kinase $3 \beta$ (GSK-3 $\beta$ ), adenomatous polyposis coli (APC) and AXIN1 or AXIN2. APC tumour suppressor gene product regulates the level of $\beta$-catenin protein by cooperating with GSK-3 $\beta$ via phosphorylation of serine/threonine residues coded on exon 3 of the $\beta$-catenin gene [21,22]. This phosphorylation is followed by degradation of $\beta$ catenin through the ubiquitin-proteasome pathway [23]. Activated Wnt signalling inhibits the phosphorylation of $\beta$-catenin, thereby preventing its degradation. The impaired $\beta$-catenin degradation leads to an increase in cytoplasmic $\beta$-catenin and its translocation to the nucleus. Nuclear $\beta$-catenin forms heterodimers with members of the TCF family of transcription factors and activates genes containing TCP-binding sites [24]. In some human cancers, mutation of either the APC gene or the $\beta$-catenin gene itself leads to the accumulation of $\beta$-catenin within the cancer cells $[21,25]$. Nuclear $\beta$ catenin is significantly associated with the invasion and metastasis of human cancers, such as carcinomas of esophagus, stomach, colon and melanomas [26,27].

The relationship between $\beta$-catenin and TAZ remains controversial. Some scholars believe that in the cytoplasm, the coactivator TAZ inhibits the CK1-mediated phosphorylation of Dvl2 by competing with Casein Kinase1 for combining with Dvl2, thereby inhibiting $\mathrm{Wnt} / \beta$-catenin signalling and promoting the degradation of $\beta$-catenin [28]. Inhibiting the expression of TAZ increases the levels of $\beta$-catenin and downstream effectors of Wnt pathway. But some are arguing that in the absence of Wnt activity, the components of the $\beta$-catenin destruction complex-APC, Axin, and GSK3-are also required to keep TAZ at low levels. TAZ degradation depends on phosphorylated $\beta$-catenin that bridges TAZ to its ubiquitin ligase $\beta$-TrCP. Upon Wnt signal, escape of $\beta$-catenin from the destruction complex impairs TAZ degradation and leads to concomitant accumulation of $\beta$-catenin and TAZ [29]. The activation of TAZ involves in Wnt signal pathway and mediates important biological effects [30]. The activated hippo pathway can reduce stability and transcriptional activity in nuclear of $\beta$-catenin by phosphorylation of YAP and TAZ [31].

Although $\beta$-catenin or TAZ has been demonstrated to correlate with poor prognosis of a variety of malignancies, however, the correlation of TAZ and $\beta$-catenin expression in AEG and the relevance of their co-expression within clinical parameters still remain unclear. Moreover, the relationship between $\beta$-catenin and TAZ remains controversial.

In this study, expression of TAZ and $\beta$-catenin was examined using immunohistochemistry on 135 AEG samples, compared with normal tissues and dysplasia samples. The correlation of TAZ and $\beta$-catenin expression and its relevance to clinicopathologic parameters were explored. Furthermore, the prognostic roles of TAZ and $\beta$-catenin in AEG were evaluated using Kaplan-Meier and Cox regression analysis. To the best of our knowledge, it is the first instance of reporting the correlation of TAZ and $\beta$-catenin expression and their clinical significance for patients with AEG.

\section{Methods}

\section{Patients and tissue samples}

The samples were obtained upon receipt of informed consent from patients undergoing surgical resection. 
The study was approved by the ethics committee of Shandong University School of Medicine (Jinan, China). Total samples comprised 135 cases of adenocarcinoma, 37 cases of normal mucosa tissues and 41 cases of dysplasia specimens which were located in EGJ. The cases were obtained from the archives of the department of Pathology at Qilu Hospital, Shandong University and collected from patients who underwent surgery between January 2006 and December 2007. The diagnosis was confirmed histologically in all cases, based mainly on examination of sections stained with H\&E. Before surgery, no patients had received drug intervention and preoperative chemotherapy. Pathological characteristics were obtained from the medical records and the original pathology reports, including age, gender, tumour differentiation, tumour size, lymph node status and invasion of serosa. AEG cases were staged by TNM classification according to the standard for oesophagogastric junction tumours of American Joint Committee on Cancer (AJCC). Curative resection procedure was performed in all AEG patients and the resection boundaries were negative both in intraoperative and routine pathological diagnosis. The AEG patients were followed up by phone call. The total period of follow-up was 1-81 months (median was 35 months). The endpoint of this study was overall survival which is defined as the period lapsing from the date of initial biopsy until death or last follow. By October 2013, 89 patients were reached the end events.

\section{Immunohistochemical stains}

Immunohistochemical stains were performed on formalin-fixed, paraffin-embedded specimens with the PV-9000 2-step plus poly-HRP anti-mouse/rabbit IgG detection system (ZSGB-Bio, Beijing, China) according to the manufacturer's instructions. Briefly, $4 \mu \mathrm{m} \mathrm{sec}-$ tions were cut and placed on glass slides, then dewaxed in xylene and rehydrated through graded alcohol. All the slides were boiled in EDTA antigen retrieval solution ( $\mathrm{pH}$ 9.0, ZSGB-Bio, Beijing, China) for $3 \mathrm{~min}$ to retrieval the antigen. Thereafter, the sections were incubated with $3 \%$ hydrogen peroxidase (reagent A) at room temperature (RT) for 10 min to block endogenous peroxidase activity. After rinsing in phosphate buffered saline (PBS, PH 7.2), incubated with normal goat serum (ZSGBBio, Beijing, China) to block any nonspecific reactions for $10 \mathrm{~min}$ at RT. Shook off excess goat serum, and then incubated the slides with primary antibodies overnight at $4{ }^{\circ} \mathrm{C}$, TAZ (bs-12367R, Bioss, Beijing, China) and $\beta$-catenin (ZM-0442, ZSGB-Bio, Beijing, China) were diluted 1:100 and 1:200 in PBS. The sections were washed with PBS and incubated with polymer helper (reagent B) for $20 \mathrm{~min}$. After rinsing in PBS, the sections were incubated with polyperoxidase-anti-mouse/rabbit IgG (reagent $\mathrm{C}$ ) for $30 \mathrm{~min}$ at room temperature and DAB (ZSGB-Bio, Beijing, China) was visualized. After rinsing in water, the sections were counterstained with hematoxylin, dehydrated, and coverslipped.

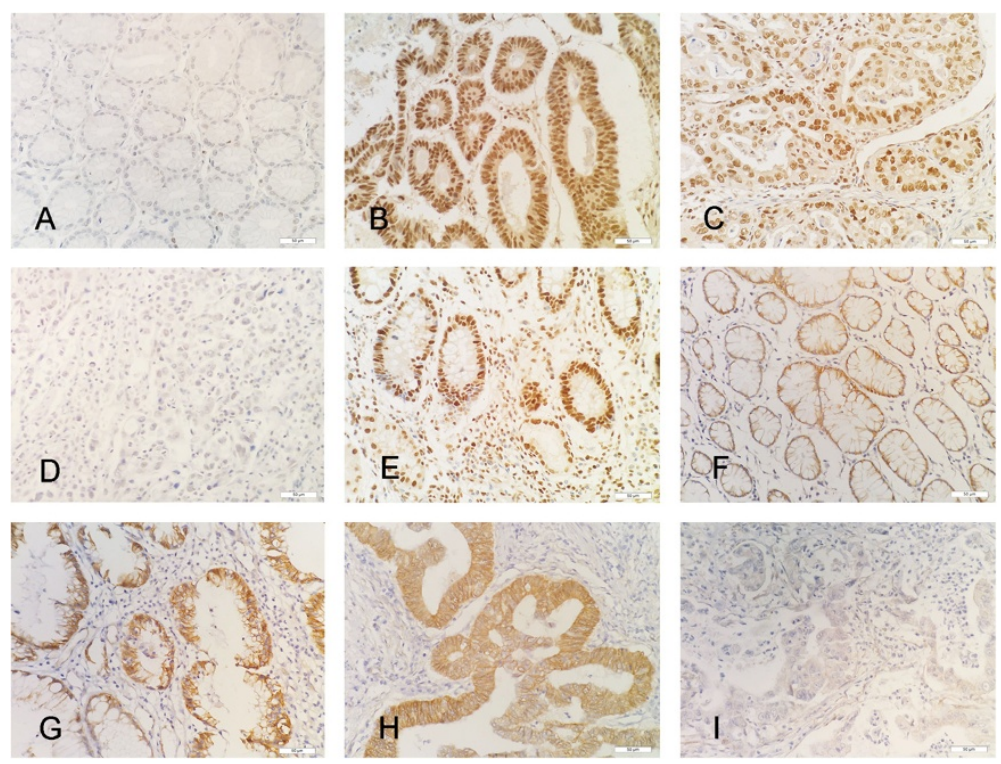

Figure 1 Expression of TAZ and $\beta$-catenin in adenocarcinoma, dysplasia and normal mucosa samples of EGJ. (A) TAZ-negative in normal mucosa. (B) TAZ-positive in dysplasia. (C) TAZ-positive in adenocarcinoma. (D) TAZ-negative in adenocarcinoma. (E) TAZ-positive in intestinal metaplasia. (F) Nuclear $\beta$-catenin negative and normal expression of membranous $\beta$-catenin in normal mucosa. (G) $\beta$-catenin- positive in dysplasia. (H) $\beta$-catenin- positive in adenocarcinoma. (I) Abnormal membranous expression of $\beta$-catenin in adenocarcinoma. (Original magnification, $\times 400)$ 
Table 1 Expression of TAZ and $\beta$-catenin proteins in different disease of EGJ

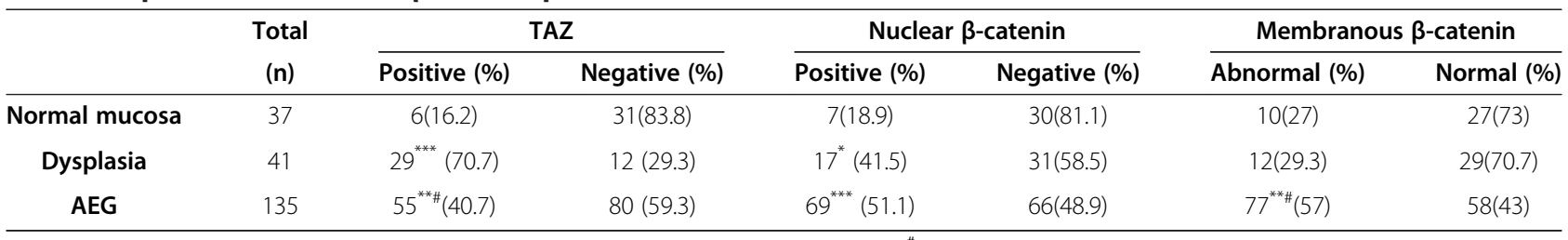

Compared with Normal mucosa: * $\mathrm{P}<0.05$, ${ }^{* *} \mathrm{P}<0.01$, ${ }^{* *} \mathrm{P}<0.001$; compared with Dysplasia: ${ }^{*} \mathrm{P}<0.01$.

\section{Positive and negative control}

Samples of AEG with high expression of TAZ and $\beta$ catenin served as the positive control. PBS was used instead of the primary antibodies as negative controls.

\section{Evaluation of immunostaining}

TAZ and $\beta$-catenin immunostaining signals were evaluated independently by two pathologists in a blinded manner. Brown nuclear staining for TAZ was considered positive/abnormal. The staining intensity of positive tumour cells was scored as 0 (no staining), 1 (weak staining), 2 (moderate staining) and 3 (strong staining). The percentage of positively stained tumour cells was scored with 5 scales: 0 (<10 \%); 1 (10\% to $25 \%) ; 2$ (26\% to $50 \%) ; 3$ (51\% to $75 \%) ; 4$ (>75\%). The final score was the product of the intensity and the percentage. For statistical reasons, a final staining score $\geq 5$ was considered to be positive. $\mathrm{Nu}$ clear and membranous $\beta$-catenin signals were evaluated independently. When more than $10 \%$ of cancer cells showed strong nuclear staining, the tumour was judged to exhibit positive/abnormal nuclear expression. In cases that demonstrated either no immunoreactivity at the membrane, or less than $10 \%$ of the tumour cells with positive membranous staining, the specimen was considered to abnormal membranous expression [32]. The percentage of positively stained cells was estimated in an average of 100 cells counted in more than 5 high-power fields $(\times 400)$.

\section{Statistical analysis}

Kruskal-Wallis text was used to assess the difference of TAZ or $\beta$-catenin expression in the three groups. The relationship between TAZ or $\beta$-catenin expression and clinicopathologic parameters were analyzed using Chi-square test and $T$ test. The correlation between TAZ and $\beta$ catenin was analyzed using the spearman's rank test. The survival curves were estimated by the Kaplan-Meier method. Log-rank test was used to compare survival curves. The HR and the 95\% CI were evaluated for each variable using the Cox univariate model. A multivariate Cox proportional hazard model was also developed using stepwise regression (forward selection) with predictive variables that were significant in the univariate analyses. $\mathrm{P}$ $<0.05$ was considered to be statistically significant. All statistical analyses were carried out using SPSS software (SPSS version 17.0 SPSS, Inc., Chicago, IL, USA).

\section{Results}

\section{Expression of TAZ and $\beta$-catenin}

As shown in Figure 1 and Table 1, a positive expression of TAZ was observed in normal mucosa $16.2 \%(6 / 37)$, dysplasia 70.7\% (29/41), and AEG 40.7\% (55/135) and the difference was significant $(\mathrm{H}=23.922, \mathrm{P}<0.001)$. The expression of TAZ in dysplasia and AEG is higher than that in normal mucosa $(\mathrm{P}<0.001,=0.008)$. And it is worth reminding that the expression of TAZ in dysplasia is higher than that in AEG ( $\mathrm{P}=0.001)$.

The positive nuclear expression rates of $\beta$-catenin in normal mucosa, dysplasia and AEG were 18.9\% (7/37), 41.5\% $(17 / 41)$ and $51.1 \%(69 / 135)$ and there was statistical difference among these groups $(\mathrm{H}=12.277, \mathrm{P}=0.002)$. The expression of $\beta$-catenin in AEG and dysplasia were significantly higher than in normal mucosa $(\mathrm{P}<0.001,=0.046)$ while no significant difference between AEG and dysplasia $(\mathrm{P}=0.276)$.

The abnormal membranous expression rates of $\beta$ catenin in the normal mucosa, dysplasia and AEG were 27\% (10/37), 29.3\% (12/41) and 57\% (77/135), respectively. There was a significant statistical difference among the groups $(\mathrm{H}=16.482, \mathrm{P}<0.001)$. Abnormal membranous expression of $\beta$-catenin in AEG was significantly higher than that in normal mucosa tissues and dysplasia $(\mathrm{P}=$ $0.001,0.002)$, while no difference was found between normal mucosa tissues and dysplasia $(\mathrm{P}=0.843)$.

In $\mathrm{AEG}$, interestingly, the statistical analysis revealed that TAZ expression positively correlated with nuclear $\beta$-catenin expression $(r=0.298$; confidence interval $(\mathrm{CI})$ 95\%, 0.136-0.462; $\mathrm{P}<0.001$ ) (Table 2). In a separate analysis, abnormal membranous expression of $\beta$-catenin is

Table 2 Relationship between the expression of TAZ and $\beta$-catenin in AEG

\begin{tabular}{lccccc}
\hline TAZ & \multicolumn{2}{c}{ Membranous $\boldsymbol{\beta}$-catenin } & & \multicolumn{2}{c}{ Nuclear $\boldsymbol{\beta}$-catenin } \\
\cline { 2 - 3 } \cline { 5 - 6 } & Normal & Abnormal & & Negative & Positive \\
\hline Negative & 41 & 39 & & 49 & 31 \\
Positive & 17 & 38 & & 17 & 38 \\
& $r=0.202 ; \mathrm{P}=0.019^{*}$ & & & $\mathrm{r}=0.298 ; \mathrm{P}<0.001^{*}$ \\
\hline
\end{tabular}

*Statistically significant. 
Table 3 Relationship between the clinicopathologic characteristics of AEG and the expression of TAZ or $\beta$-catenin

\begin{tabular}{|c|c|c|c|c|c|c|c|c|c|c|c|c|c|c|c|}
\hline \multirow{2}{*}{$\begin{array}{l}\text { Clinicopathologic } \\
\text { characteristics }\end{array}$} & \multicolumn{2}{|c|}{ TAZ } & \multirow[t]{2}{*}{$\mathbf{P}$} & \multicolumn{2}{|c|}{$\beta$-catenin (nuclear) } & \multirow[t]{2}{*}{$\mathbf{P}$} & \multicolumn{2}{|c|}{$\beta$-catenin (membranous) } & \multirow[t]{2}{*}{$\mathbf{P}$} & \multicolumn{2}{|c|}{ TAZ \& $\beta$-catenin (nuclear) } & \multirow[t]{2}{*}{$\mathbf{P}$} & \multicolumn{2}{|c|}{ TAZ \& $\beta$-catenin (membranous) } & \multirow[t]{2}{*}{$\mathbf{P}$} \\
\hline & Negative & Positive & & Negative & Positive & & Normal & Abnormal & & Others & Positive & & Others & Positive & \\
\hline \multicolumn{16}{|l|}{ Gender } \\
\hline Male & 64 & 46 & 0.593 & 52 & 58 & 0.431 & 45 & 65 & 0.312 & 79 & 31 & 0.99 & 64 & 46 & 0.842 \\
\hline Female & 16 & 9 & & 14 & 11 & & 13 & 12 & & 18 & 7 & & 14 & 11 & \\
\hline \multicolumn{16}{|l|}{ Age } \\
\hline$<60$ years old & 26 & 22 & 0.371 & 13 & 35 & 0.001 & 21 & 27 & 0.891 & 31 & 17 & 0.163 & 22 & 26 & 0.037 \\
\hline$\geq 60$ years old & 54 & 33 & & 53 & 34 & & 37 & 50 & & 66 & 21 & & 56 & 31 & \\
\hline \multicolumn{16}{|l|}{ Invasion of serosa } \\
\hline Positive & 21 & 38 & 0.001 & 21 & 38 & 0.006 & 18 & 41 & 0.010 & 31 & 28 & 0.001 & 25 & 34 & 0.001 \\
\hline Negative & 59 & 17 & & 45 & 31 & & 40 & 36 & & 66 & 10 & & 53 & 23 & \\
\hline \multicolumn{16}{|l|}{ lymphatic metastasis } \\
\hline Positive & 34 & 42 & 0.001 & 30 & 46 & 0.013 & 27 & 49 & 0.048 & 43 & 33 & 0.001 & 37 & 39 & 0.015 \\
\hline Negative & 46 & 13 & & 36 & 23 & & 31 & 28 & & 54 & 5 & & 41 & 18 & \\
\hline \multicolumn{16}{|l|}{ Differentiation } \\
\hline Well-differentiated & 51 & 22 & 0.007 & 43 & 30 & 0.012 & 47 & 26 & 0.001 & 59 & 14 & 0.012 & 57 & 16 & 0.001 \\
\hline Poorly differentiated & 29 & 33 & & 23 & 39 & & 11 & 51 & & 38 & 24 & & 21 & 41 & \\
\hline \multicolumn{16}{|l|}{ TNM stage } \\
\hline Stage $0 \mid \|$ & 55 & 24 & 0.004 & 43 & 36 & 0.126 & 39 & 40 & 0.074 & 65 & 14 & 0.001 & 65 & 32 & 0.001 \\
\hline Stage III IV & 25 & 31 & & 23 & 33 & & 19 & 37 & & 32 & 24 & & 14 & 24 & \\
\hline
\end{tabular}


also correlated with expression of TAZ $(r=0.202$; CI 95\%, 0.041-0.357; $\mathrm{P}=0.019$ ).

\section{Association of TAZ and $\beta$-catenin expression with clinico- pathological characteristics}

As shown in Table 3, the abnormal expression of TAZ, nuclear $\beta$-catenin, membranous $\beta$-catenin, TAZ \& nuclear $\beta$-catenin and TAZ \& membranous $\beta$-catenin were markedly correlated with lymph node metastasis, invasion of serosa and tumour differentiation $(\mathrm{P}<0.05$, respectively). In addition, the abnormal expression of nuclear $\beta$-catenin and TAZ \& membranous $\beta$-catenin were positively correlated with age $(\mathrm{P}<0.001,=0.037)$. The abnormal expression of TAZ, membranous $\beta$-catenin, TAZ \& nuclear
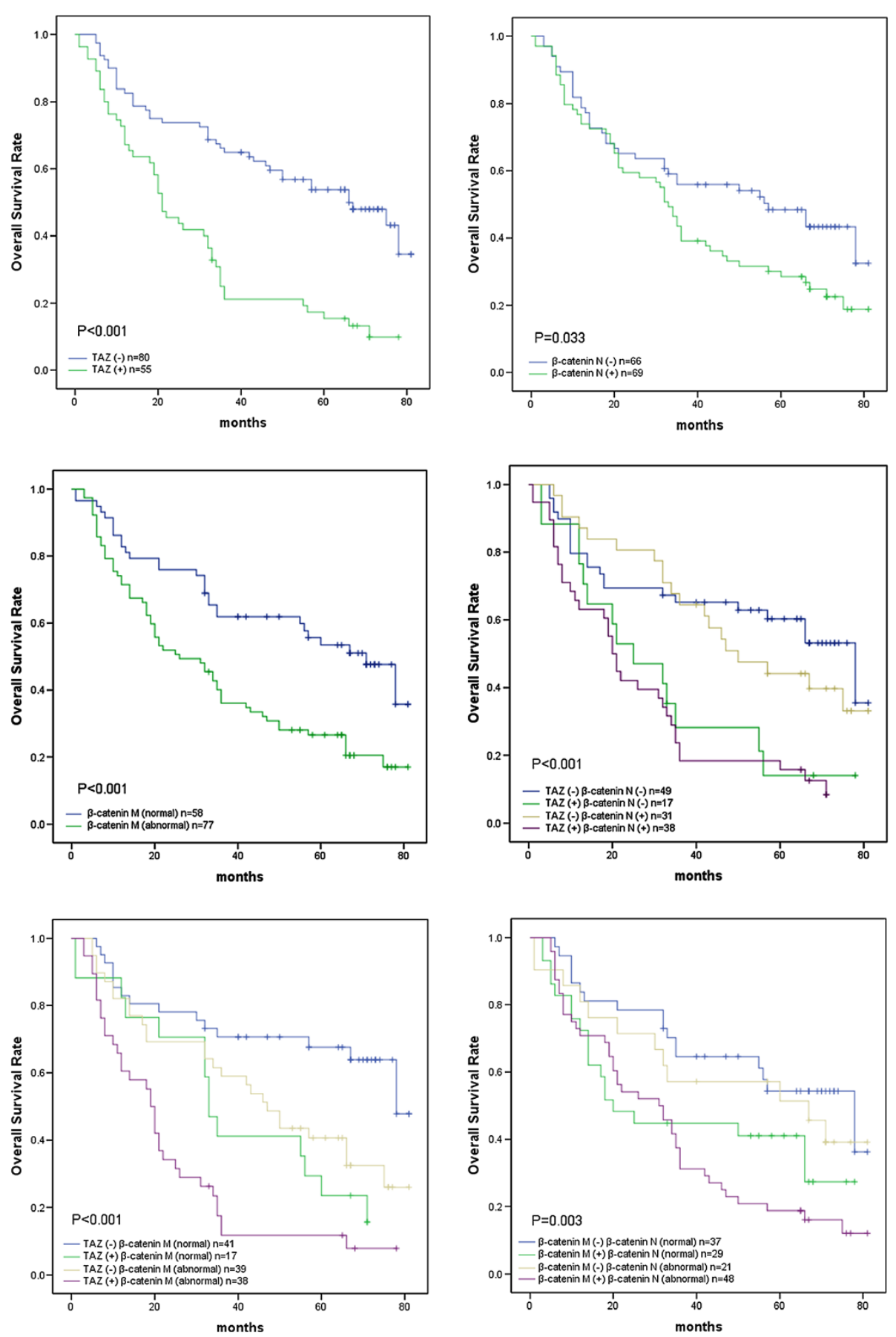

Figure 2 Kaplan-Meier analysis of overall survival rates in 135 AEG patients. $\beta$-catenin $N$, nuclear expression of $\beta$-catenin. $\beta$-catenin $M$, membranous expression of $\beta$-catenin. 
$\beta$-catenin and TAZ \& membranous $\beta$-catenin were markedly correlated with TNM stages. There was no significant association of TAZ or $\beta$-catenin expression with other clinicopathological features mentioned in the table.

No significant correlations were found between expression of TAZ or $\beta$-catenin and tumour size. The tumour size of TAZ positive cases was $4.74 \pm 1.872$ centimeter, the negative one was $5.03 \pm 1.923$ centimeter, $\mathrm{P}=0.392$. The tumour sizes of nuclear $\beta$-catenin positive was $4.92 \pm 2.019$ centimeter and the negative one was $4.79 \pm 1.686$ centimeter, $\mathrm{P}=0.685$. The tumour sizes of abnormal expression membranous $\beta$-catenin was $4.89 \pm$ 1.758 centimeter and the normal one was $4.83 \pm 2.059$ centimeter, $\mathrm{P}=0.863$.

\section{Association of TAZ and $\beta$-catenin expression with OS}

When the AEG cases were analyzed by Kaplan-Meier curves (Figure 2), we observed that the abnormal expression of TAZ, $\beta$-catenin (nuclear and membranous), nuclear $\beta$-catenin, membranous $\beta$-catenin, TAZ \& nuclear $\beta$-catenin and TAZ \& membranous $\beta$-catenin significantly affected overall survival rate of AEG patients $(\mathrm{P}<$ 0.05 for all). Patients with abnormal expression tumour exhibited a shorter OS than those with normal one. Moreover, patients with both TAZ and $\beta$-catenin (nuclear and membranous) abnormal expression exhibited the worst survival (mean of 21.2 \pm 3.0 months and median of 19 months). On the contrary, patients with both normal expression demonstrated the best overall survival (mean of $63.4 \pm 5.2$ months and median of 78 months, $\mathrm{P}<0.001$, Figure 3). As summarized in Table 4, the Cox univariate model highlighted that abnormal expression of TAZ, nuclear $\beta$-catenin, membranous $\beta$-catenin, TAZ $\&$ nuclear $\beta$-catenin, TAZ \& membranous $\beta$-catenin, TAZ \& $\beta$-catenin (nuclear and membranous) tumour differentiation and nodal status significantly impact overall survival time ( $\mathrm{P}<0.05$ for all). More importantly, multivariate analysis revealed that tumour differentiation (Hazard rate (HR) 1.870, CI 95\%, 1.216-2.877, $\mathrm{P}=0.004$ ), TAZ expression (HR 1.879, CI 95\%, 1.079-3.218, P = 0.022 ) and abnormal expression of TAZ \& $\beta$-catenin (nuclear and membranous) (HR 1.899, CI 95\%, 1.0533.423, $\mathrm{P}=0.033$ ) were independent negative prognostic variables influencing $\mathrm{OS}$.

\section{Discussion}

AEG includes adenocarcinomas of distal esophageal, cardia and proximal gastric and has been regarded as a separate entity because it appears to have distinct features. Proximal gastric cancer, which is similar with distal esophageal adenocarcinoma, is very different from distal gastric cancer on patient's age, gender, incidence, tumour biological behaviors and clinicopathological features [33]. Lower thoracic esophageal carcinoma is more likely to

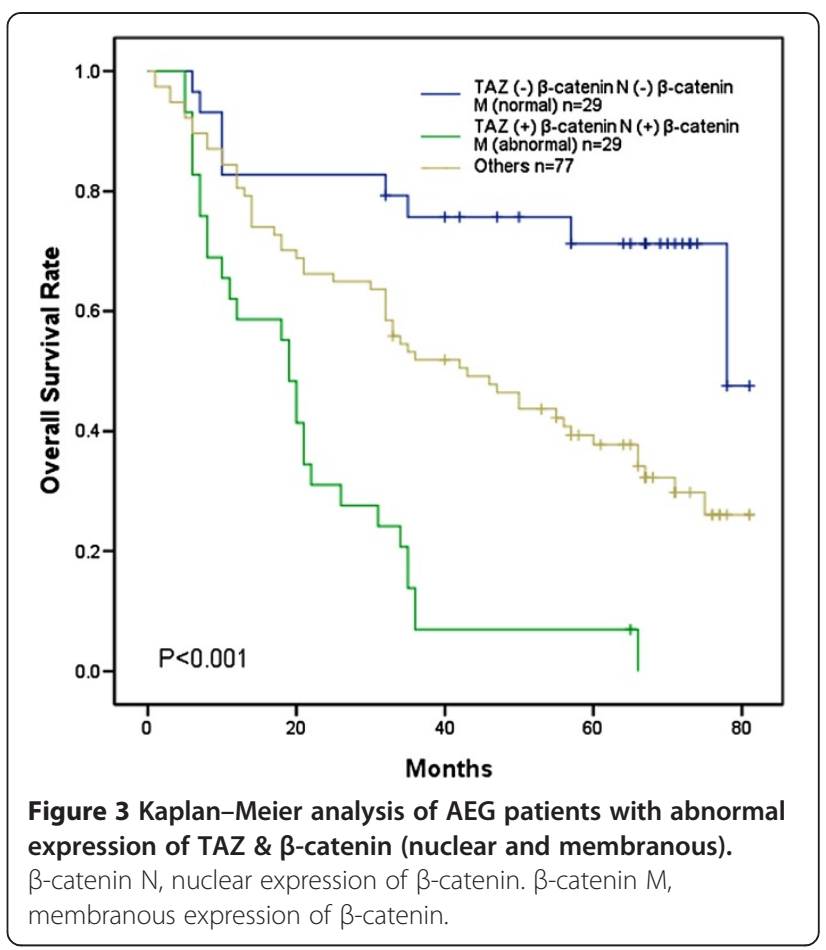

have lymph node metastasis, fast proliferation and poor prognosis than the upper or middle one. So the concept of AEG was put forward as a special entity. The incidence of AEG is going higher than that of distal stomach cancer [34]. The possible causes of AEG and gastric cancer are different. Notably, it has been demonstrated that H. pylori infection is one of the clear risk factors to gastric cancer, while some research suggests that negative $H$. pylori status correlates with a poor prognosis with AEG $[35,36]$. Gastric cancers located in the distal stomach are associated with better prognosis than those located in the proximal region [37]. Differences in sex distinction and mean age were found between AEG and the distal gastric cancer [38]. In our study, the ratio of man to woman was 4.4 to 1 , and the average age was 62.6 years old, and patients of 60 years old and above were accounted for 64.4 percent of AEG patients, which were consistent with previous studies. The rate of lymph node metastasis was $56.3 \%$, and associated with over expression of TAZ or $\beta$-catenin. Some earlier researches produced similar findings: $\beta$-catenin was over expressed in metastatic sentinel lymph node, and strongly associated with liver metastasis [39]. The percentage of well differentiated carcinoma in AEG (49.5\%) was higher than that in other malignant tumours [40].

The accumulated evidence from basic and clinical researches indicates that both TAZ and $\beta$-catenin were correlated with tumour invasion, metastasis, and poor prognosis in human cancers $[21,26,27,41,42]$. However, the regulation of TAZ upon $\beta$-catenin expression and the relevance of their expression to clinical pathological 
Table 4 Cox proportional hazard regression model analysis

\begin{tabular}{|c|c|c|c|c|}
\hline \multirow[t]{2}{*}{ Factors } & \multicolumn{2}{|c|}{ Univariate analysis } & \multicolumn{2}{|c|}{ Multivariate analysis } \\
\hline & $\mathrm{HR}(95 \% \mathrm{Cl})$ & P-value & $\mathrm{HR}(95 \% \mathrm{Cl})$ & P-value \\
\hline \multicolumn{5}{|l|}{ TAZ } \\
\hline Positive vs. Negative & $2.740(1.791-4.192)$ & $<0.001^{*}$ & $1.879(1.097-3.218)$ & $0.022^{*}$ \\
\hline \multicolumn{5}{|l|}{$\beta$-catenin (nuclear) } \\
\hline Positive vs. Negative & $1.572(1.028-2.402)$ & $0.037^{*}$ & & \\
\hline \multicolumn{5}{|l|}{$\beta$-catenin (membranous) } \\
\hline Abnormal vs. Normal & $2.153(1.377-3.367)$ & $<0.001^{*}$ & & \\
\hline \multicolumn{5}{|l|}{ TAZ \& $\beta$-catenin (nuclear) } \\
\hline Positive vs. All others & $2.482(1.606-3.837)$ & $<0.001^{*}$ & & \\
\hline \multicolumn{5}{|c|}{ TAZ \& $\beta$-catenin (membranous) } \\
\hline Abnormal vs. All others & $3.458(1.732-6.908)$ & $<0.001^{*}$ & & \\
\hline \multicolumn{5}{|c|}{ TAZ \& $\beta$-catenin (nuclear and membranous) } \\
\hline Abnormal vs. All others & $3.330(2.082-5.328)$ & $<0.001^{*}$ & $1.899(1.053-3.423)$ & $0.033^{*}$ \\
\hline \multicolumn{5}{|l|}{ Tumor size (diameter) } \\
\hline$\geq 3 \mathrm{~cm}$ vs. $<3 \mathrm{~cm}$ & $1.490(0.886-2.506)$ & 0.132 & & \\
\hline \multicolumn{5}{|l|}{ Lymph node status } \\
\hline Positive vs. Negative & $1.548(1.006-2.383)$ & $0.047^{*}$ & & \\
\hline \multicolumn{5}{|l|}{ Tumor differentiation } \\
\hline Poorly vs. Well-differentiated & $2.165(1.419-3.304)$ & $<0.001^{*}$ & $1.870(1.216-2.877)$ & $0.004^{*}$ \\
\hline \multicolumn{5}{|l|}{ Age (years) } \\
\hline$\geq 60$ vs. $<60$ & $1.216(0.782-1.890)$ & 0.386 & & \\
\hline \multicolumn{5}{|l|}{ Gender } \\
\hline Male vs. Female & $1.687(0.918-3.102)$ & 0.092 & & \\
\hline \multicolumn{5}{|l|}{ Invation of serosa } \\
\hline Positive vs. Negative & $1.355(0.893-2.057)$ & 0.154 & & \\
\hline
\end{tabular}

*Statistically significant.

features in AEG were still unknown. In this study, we examined the expression of TAZ and $\beta$-catenin in 135 AEG specimens using immunohistochemistry stain, and compared with expressions in normal mucosa and dysplasia samples. To our knowledge, it is the first time that clinical evidence has been provided to indicate that TAZ and $\beta$-catenin expression were positively correlated in AEG.

TAZ is a WW domain containing transcriptional coactivator that modulates cell differentiation and development of multiple organs [7]. TAZ promotes cell proliferation and epithelial-mesenchymal transition and plays an important role in tumourigenesis [43]. Zhao et al. reported that TAZ can promote cell growth in breast partially through up-regulating KLF5 protein and enhancing its activity and protecting it from WWP1mediated degradation [44]. There was also a report that TAZ induces growth factor-independent proliferation through activation of EGFR ligand amphiregulin [45]. Here we found that TAZ protein was strictly located in the nucleus of AEG cells using IHC staining, and the expression level of TAZ was significantly upregulated in AEG and dysplasia than that in normal mucosa. Moreover, we also observed that the expression of TAZ was positive in intestinal metaplasia of EGJ (Figure 1E). These results suggest that TAZ upregulation is an early event in the progression of AEG.

$\beta$-catenin plays an essential role in the regulation of the E-cadherin-catenin cell adhesion complex as well as in the Wnt signal pathway [20]. It has been reported that $\beta$-catenin was significantly associated with the invasion and metastasis of carcinomas of the esophagus, stomach, colon, liver and melanomas $[21,26,27,46]$. In this study, we evaluated nuclear and membranous $\beta$-catenin signals respectively in immunohistochemical stain. Nuclear $\beta$ catenin expression in three groups was similar with TAZ, indicating that nuclear $\beta$-catenin accumulation was involved in the carcinogenesis and tumour development of AEG. While abnormal membranous expression rate of $\beta$-catenin in AEG was higher than that in normal mucosa and dysplasia, no difference between normal mucosa and dysplasia. Furthermore, abnormal expression of 
membranous $\beta$-catenin was correlated with lymph node matastisis and invasion of serosa. We can infer that the decreased expression of membranous $\beta$-catenin may occur in later stage of AEG and associated with invasion and metastasis.

In our study, the abnormal expression of TAZ, nuclear $\beta$-catenin, membranous $\beta$-catenin, TAZ \& nuclear $\beta$ catenin and TAZ \& membranous $\beta$-catenin were positively correlated with lymph node metastasis, invasion of serosa and tumour differentiation which are crucial histological features associated with poor prognosis, and these findings were consistent with previous results. Because TNM classification is closely related to lymph node status, tumour invasion and metastasis, the abnormal expression of TAZ, membranous $\beta$-catenin, TAZ \& nuclear $\beta$-catenin and TAZ \& membranous $\beta$-catenin were also markedly correlated with TNM stages. We believe that the abnormal TAZ or $\beta$-catenin expression in tumour cells promotes tumour cell EMT and, therefore, facilitates tumour cell migration and metastasis into the lymphatic vessels.

In this study over expression of TAZ and nuclear expression of $\beta$-catenin were positively correlated, which was consistent with the previous findings [29-31]. We can infer that except for acting as a transcription coactivator, TAZ may play a key role in suppressing the degradation of $\beta$-catenin and promoting $\beta$-catenin to enter nuclear region. We also found that the expression of TAZ was positively associated with decreased membranous expression of $\beta$-catenin. However, the molecular mechanism underlying this expression pattern and its clinical importance need future investigation. One possible mechanism is TAZ affects cadherin-mediated cell-cell adhesion system by regulating membranous expression of $\beta$-catenin.

Previously reported that colorectal cancer patients with higher TAZ expression showed a trend of shorter survival times [42]. Consistently, in our study the Kaplan-Meier survival analysis revealed that patients with abnormal expression of TAZ or $\beta$-catenin (nuclear or membranous) expression showed worse OS than those with normal TAZ or $\beta$-catenin expression. Further still, patients with abnormal expression of both TAZ and $\beta$-catenin (nuclear and membranous) exhibited the worst overall survival. On the contrary, patients with both normal results demonstrated the best survival. These results indicate that combining TAZ and $\beta$-catenin predicts worse survival and may serve as the key molecular prognostic indicator for AEG patient survival.

Consistent with previous reports, our Cox multivariate analysis demonstrated that high TAZ expression levels and combined abnormal expression of TAZ \& $\beta$-catenin (nuclear and membranous) were independent negative prognostic factors together with tumour differentiation.
The result strongly suggests that TAZ and $\beta$-catenin may serve as disease prognosis indicators for AEG patient survival. Moreover, the regulation of TAZ on $\beta$-catenin expression provides a new molecular mechanism underlying TAZ promoted AEG progression and metastasis and may indeed suggest a feasible therapeutic strategy to inhibit AEG metastasis by targeting TAZ and $\beta$-catenin expressions.

\section{Conclusions}

In conclusion, our research demonstrated that over expression of TAZ was associated with abnormal expression of $\beta$-catenin in AEG. We also provided convincing evidence that abnormal expression of TAZ or $\beta$-catenin was correlated with the poor prognosis of patients with AEG. Moreover, abnormal expression of TAZ and TAZ $\& \beta$-catenin (nuclear and membranous) could be independent predictors of prognosis in AEG, so targeting TAZ and $\beta$-catenin could prove to be a promising therapeutic strategy for the treatment of AEG.

\section{Abbreviations}

AEG: Adenocarcinoma of the esophagogastric junction; EGJ: Esophagogastric junction; TAZ: Transcriptional coactivator with PDZ-binding motif;

$\mathrm{Cl}$ : Confidence interval; HR: Hazard ratio; OS: Overall survival.

\section{Competing interests}

The authors declared that they have no competing interests.

\section{Authors' contributions}

ZJ conceived of the study. SL participated in experiments, performed the statistical analysis and drafted the manuscript. CF and SW participated in case selection and followed up with AEG patients. All authors scored the immunostaining, read and approved the final manuscript.

\section{Acknowledgements}

This study was supported by grants from Natural Science Foundation of Shandong Province (ZR2011HM075) and the Foundation for Outstanding Young Scientist in Shandong Province (BS2012YY020)

\section{Author details}

'Department of Pathology, Shandong University School of Medicine, 44\#, Wenhua Xi Road, Jinan, Shandong 250012, People's Republic of China. ${ }^{2}$ Department of Pathology, Qilu Hospital, Shandong University, 107\#,Wenhua Xi Road, Jinan, Shandong 250012, People's Republic of China. ${ }^{3}$ Department of Gastroenterology, Qilu Hospital, Shandong University, 107\#,Wenhua Xi Road, Jinan, Shandong 250012, People's Republic of China. ${ }^{4}$ Department of Orthopedics, Qilu Hospital, Shandong University, 107\#,Wenhua Xi Road, Jinan, Shandong 250012, People's Republic of China. ${ }^{5}$ Department of Gynecology and Obstetrics, Laiyang City Centre Hospital, Laiyang, Shandong 265200, People's Republic of China.

\section{Received: 23 April 2014 Accepted: 22 June 2014}

Published: 16 July 2014

\section{References}

1. Siewert JR, Stein HJ: Adenocarcinoma of the gastresopha-geal junction: classifiction, pathology and extent of resection. Dis Esoph 1996, 9:173-182.

2. Oda I, Abe S, Kusano C, Suzuki H, Nonaka S, Yoshinaga S, Taniguchi H, Shimoda T, Gotoda T: Correlation between endoscopic macroscopic type and invasion depth for early esophagogastric junction adenocarcinomas. Gastric Cancer 2011, 14:22-27. 
3. Abdi-Rad A, Ghaderi-sohi S, Nadimi-Barfroosh H, Emami S: Trend in incidence of gastric adenocarcinoma by tumour location from 1969-2004: a study in one referral center in Iran. Diagn Pathol 2006, 1:5. 4. Warneke VS, Behrens HM, Hartmann JT, Held H, Becker T, Schwarz NT, Röcken C: Cohort study based on the seventh edition of the TNM classification for gastric cancer: proposal of a new staging system. J Clin Oncol 2011, 29:2364-2371

5. Feith M, Stein HJ, Siewert JR: Adenocarcinoma of the esophagogastric junction: surgical therapy based on 1602 consecutive resected patients. Surg Oncol Clin N Am 2006, 15:751-764.

6. Hosokawa Y, Kinoshita T, Konishi M, Takahashi S, Gotohda N, Kato Y, Daiko $\mathrm{H}$, Nishimura M, Katsumata K, Sugiyama Y, Kinoshita T: Clinicopathological features and prognostic factors of adenocarcinoma of the esophagogastric junction according to Siewert classification: experiences at a single institution in Japan. Ann Surg Oncol 2012, 19:677-683.

7. Kanai F, Marignani PA, Sarbassova D, Yagi R, Hall RA, Donowitz M, Hisaminato A, Fujiwara T, Ito Y, Cantley LC, Yaffe MB: TAZ: a nove transcriptional co-activator regulated by interactions with14-3-3 and PDZ domain proteins. EMBO J 2000, 19:6778-6791.

8. Zhang L, Yue T, Jiang J: Hippo signaling pathway and organ size control Fly (Austin) 2009, 3:68-73.

9. Zhao B, Lei QY, Guan KL: The Hippo-YAP path-way: new connections between regulation of organ size and cancer. Curr Opin Cell Biol 2008 20:638-646.

10. Wu S, Huang J, Dong J, Pan D: Hippo encodes a Ste-20 family protein kinase that restricts cell proliferationand promotes apoptosis in conjunction with salvador and warts. Cell 2003, 114:445-456.

11. Justice RW, Zilian O, Woods DF, Noll M, Bryant PJ: The Drosophila tumour suppressor gene warts encodes a homolog of humanmyotonic dystrophy kinase and is required for the control of cell shape and proliferation. Genes Dev 1995, 9:534-546.

12. Kango-Singh $M$, Nolo $R$, Tao $C$, Verstreken $P$, Hiesinger $P R$, Bellen $H J$, Halder G: Shar-pei mediates cell proliferation arrest during imaginal disc growth inDrosophila. Development 2002, 129:5719-5730.

13. Dong J, Feldmann G, Huang J, Wu S, Zhang N, Comerford SA, Gayyed MF, Anders RA, Maitra A, Pan D: Elucidation of a universal size-control mechanism in Drosophila and mammals. Cell 2007, 130:1120-1133.

14. Cui CB, Cooper LF, Yang X, Karsenty G, Aukhil I: Transcriptional coactivation of bone-specific transcription factor Cbfa1 by TAZ. Mol Cell Biol 2003, 23:1004-1013.

15. Murakami M, Tominaga J, Makita R, Uchijima $Y$, Kurihara $Y$, Nakagawa $O$, Asano T, Kurihara H: Transcriptional activity of Pax3 is co-activated by TAZ. Biochem Biophys Res Commun 2006, 339:533-539.

16. Mahoney WM Jr, Hong JH, Yaffe MB, Farrance IK: The transcriptional co-activator TAZ interacts differentially withtranscriptional enhancer factor-1 (TEF-1) family members. Biochem J 2005, 388:217-225.

17. Hong JH, Hwang ES, McManus MT, Amsterdam A, Tian Y, Kalmukova R, Mueller E, Benjamin T, Spiegelman BM, Sharp PA, Hopkins N, Yaffe MB: TAZ, a transcriptional modulator of mesenchymal stem cell differentiation. Science 2005, 309:1074-1078.

18. Hong JH, Yaffe MB: TAZ: a beta-catenin-like molecule that regulates mesenchymal stem cell differentiation. Cell Cycle 2006, 5:176-179.

19. Murakami M, Nakagawa M, Olson EN, Nakagawa O: A WW domain protein TAZ is a critical coactivator for TBX5, a transcription factor implicated in Holt-Oram syndrome. Proc Natl Acad Sci U S A 2005, 102:18034-18039.

20. McCrea PD, Gumbiner BM: Purification of a 92-kDa cytoplasmic protein tightly associated with the cell-cell adhesion molecule E-cadherin (uvomorulin). Characterization and extractability of the protein complex from the cell cytostructure. J Biol Chem 1991, 266:4514-4520

21. Rubinfeld B, Robbins P, El-Gamil M, Albert I, Porfiri E, Polakis P: Stabilization of beta-catenin by genetic defects in melanoma cell lines. Science 1997, 275:1790-1792.

22. Munemitsu S, Albert I, Souza B, Rubinfeld B, Polakis P: Regulation of intracellular beta-catenin levels by the adenomatous polyposis coli (APC) tumour-suppressor protein. Proc Natl Acad Sci U S A 1995, 92:3046-3050.

23. Orford K, Crockett C, Jensen JP, Weissman AM, Byers SW: Serine phosphorylation-regulated ubiquitination and degradation of betacatenin. J Biol Chem 1997, 272:24735-24738.

24. Bienz M, Clevers H: Linking colorectal cancer to Wnt signaling. Cell 2000, 103:311-320.
25. Iwao K, Nakamori S, Kameyama M, Imaoka S, Kinoshita M, Fukui T, Ishiguro S, Nakamura Y, Miyoshi Y: Activation of the beta-catenin gene by interstitial deletions involving exon 3 in primary colorectal carcinomas without adenomatous polyposis coli mutations. Cancer Res 1998, 58:1021-1026.

26. Osterheld MC, Bian YS, Bosman FT, Benhattar J, Fontolliet C: Beta-catenin expression and its association with prognostic factors in adenocarcinoma developed in Barrett esophagus. Am J Clin Pathol 2002, 117:451-456.

27. Kim HS, Hong EK, Park SY, Kim WH, Lee HS: Expression of beta-catenin and E-cadherin in the adenoma-carcinoma sequence of the stomach. Anticancer Res 2003, 23:2863-2868.

28. Varelas X, Miller BW, SoPko R, Song S, Gregorieff A, Fellouse FA, Sakuma R, Pawson T, Hunziker W, McNeill H, Wrana JL, Attisano L: The Hippo pathway regulates Wnt/beta-catenin signaling. Dev Cell 2010, 18:579-591.

29. Azzolin L, Zanconato F, Bresolin S, Forcato M, Basso G, Bicciato S, Cordenonsi M, Piccolo S: Role of TAZ as mediator of Wnt signaling. Cell 2012, 151:1443-1456.

30. Tsai BP, Hoverter NP, Waterman ML: Blending hippo and WNT: sharing messengers and regulation. Cell 2012, 151:1401-1403.

31. Imajo M, Miyatake K, limura A, Miyamoto A, Nishida E: A molecular mechanism that links Hippo sig-nalling to the inhibition of Wnt/ $\beta$-catenin signalling. EMBO J 2012, 31:1109-1122.

32. Woo DK, Kim HS, Lee HS, Kang YH, Yang HK, Kim WH: Altered expression and mutation of beta-catenin gene in gastric carcinomas and cell lines. Int J Cancer 2001, 95:108-113.

33. Shan L, Ying J, Lu N: HER2 expression and relevant clinicopathological features in gastric and gastroesophageal junction adenocarcinoma in a Chinese population. Diagn Pathol 2013, 8:76.

34. Hasegawa S, Yoshikawa T: Adenocarcinoma of the esophagogastric junction: incidence, characteristics, and treatment strategies. Gastric Cancer 2010, 13:63-73.

35. Meimarakis G, Winter H, Assmann I, Kopp R, Lehn N, Kist M, Stolte M, Jauch KW, Hatz RA: Helicobacter pylori as a prognostic indicator after curative resection of gastric carcinoma: a prospective study. Lancet Oncol 2006, 7:211-222.

36. Marrelli D, Pedrazzani C, Berardi A, Corso G, Neri A, Garosi L, Vindigni C, Santucci A, Figura N, Roviello F: Negative Helicobacter pylori status is associated with poor prognosis in patients with gastric cancer. Cancer 2009, 115:2071-2080.

37. Hundahl SA, Phillips JL, Menck HR: The National cancer Data Base Report on poor survival of U.S. gastric carcinoma patients treated with gastrectomy: fifth edition American Joint Committee on Cancer staging, proximal disease, and the "different disease" hypothesis. Cancer 2000, 88:921-932.

38. Hasegawa S, Yoshikawa T, Cho H, Tsuburaya A, Kobayashi O: Is adenocarcinoma of the esophagogastric junction different between Japanand western countries? The incidence and clinicopathological features at aJapanese high-volume cancer center. World J Surg 2009, 33:95-103.

39. Hongxia C, Hui L, Yejun Q, Ying L: Nuclear beta-catenin overexpression in metastatic sentinel lymph node is associated with synchronous liver metastasis in colorectal cancer. Diagn Pathol 2011, 6:109.

40. Leers JM, DeMeester SR, Chan N, Ayazi S, Oezcelik A, Abate E, Banki F, Lipham JC, Hagen JA, DeMeester TR: Clinical characteristics, biologic behavior, and survival after esophagectomyare similar for adenocarcinoma of the gastroesophageal junction and thedistal esophagus. J Thorac Cardiovasc Surg 2009, 138:594-602.

41. Jeong GO, Shin SH, Seo EJ, Kwon YW, Heo SC, Kim KH, Yoon MS, Suh DS, Kim JH: TAZ mediates lysophosphatidic acid-induced migration and proliferation of epithelial ovarian cancer cells. Cell Physiol Biochem 2013, 32:253-263

42. Wang L, Shi S, Guo Z, Zhang X, Han S, Yang A, Wen W, Zhu Q: Overexpression of YAP and TAZ is an independent predictor of prognosis in colorectal cancer and related to the proliferation and metastasis of colon cancer cells. PLoS One 2013, 8:e65539.

43. Lei OY, Zhang $H$, Zhao B, Zha ZY, Bai F, Pei XH, Zhao S, Xiong Y, Guan KL: TAZ promotes cell proliferation and epithelial-mesenchymal transition and is inhibited by the hippopathway. Mol Cell Biol 2008, 28:2426-2436.

44. Zhao D, Zhi X, Zhou Z, Chen C: TAZ antagonizes the WWP1-mediated KLF5 degradation and promotes breast cell proliferation and tumourigenesis. Carcinogenesis 2012, 33:59-67. 
45. Yang N, Morrison CD, Liu P, Miecznikowski J, Bshara W, Han S, Zhu Q, Omilian AR, Li X, Zhang J: TAZ induces growth factor-independent proliferation through activation of EGFR ligand amphiregulin. Cell Cycle 2012, 11:2922-2930.

46. Mao TL, Chu JS, Jeng YM, Lai PL, Hsu HC: Expression of mutant nuclear beta-catenin correlates with non-invasive hepatocellular carcinoma, absence of portal vein spread, and good prognosis. J Pathol 2001, 193:95-101.

doi:10.1186/1746-1596-9-125

Cite this article as: Sun et al:: Prognostic impact of TAZ and $\beta$-catenin expression in adenocarcinoma of the esophagogastric junction.

Diagnostic Pathology 2014 9:125.

Submit your next manuscript to BioMed Central and take full advantage of:

- Convenient online submission

- Thorough peer review

- No space constraints or color figure charges

- Immediate publication on acceptance

- Inclusion in PubMed, CAS, Scopus and Google Scholar

- Research which is freely available for redistribution

Submit your manuscript at www.biomedcentral.com/submit 